lary. 'The calvarium, which is somewhat collapsed, is filled with a caseous mass. The whole is covered by a closely adherent thin fibrous tissue. The specimen is now in the museum of Rush Medical College.

\section{TWO CASES OF ECTOPIC GESTATION.}

\section{E. E. GELDER, M.D. \\ PEORIA, ILL.}

As tubal pregnancy must often times be diagnosed from the history rather than by physical findings, a complete history of each case should be reported. Each specimen removed at operation or autopsy should be studied to ascertain, if possible, the cause of failure of the impregnated ovum to descend the tube and enter the uterus. In pursuance of these ideas I make this report.

CASE 1.-In July 1906, I saw this woman at her home, in consultation with her physician, Dr. W. H. Willis of this city, and obtained the following history :

History.-Mrs. -, aged 30, had been married nine years but had not been pregnant previously. Her menstrual periods had always been regular, not painful, and the flow normal in quantity and duration. She never had intermenstrual bleeding or leucorrhea, and never had symptoms referable to inflammatory or infectious disease or any of the pelvic viscera. In February, 1906, she consulted Dr. Willis for relief from sterility. Under anesthesia he dilated the cervix; nothing abnormal was removed by the curette. She menstruated in May but missed in June. Early in July, two weeks after her period was due, she began to flow a little; a week later she developed pain in the uterus and apparently had an early miscarriage but did not save what passed. After this she had no more uterine pain but pain and tenderness in the left side of the pelvis. About July 14, or one week after the supposed miscarriage, she was taken with sudden severe pain in the left side with some collapse; although the severity of the pain lessened, it never left her entirely.

Examination.-On July 27, under anesthesia, we found the uterus approximating the normal position with the exception of the cervix which was slightly displaced to the right; the cavity of the uterus was empty and of normal length. The left broad ligament was filled with a semi-solid mass which also extended into the posterior cul-de sac.

Diagnosis.-Ectopic gestation with rupture of the tube within the broad ligament.

Operation.-The following day, July 28, I assisted Dr. Willis in operating on the patient. Incision proved our diagnosis correct; a blood clot entirely filled the left broad ligament and had dissected across beneath its posterior layer to beyond the median line. The clots together with the left tube and ovary were removed; the broad ligament was washed out, and the two layers stitched together. The right tube and ovary were normal. The wound was closed without drainage and the patient made a rapid and uneventful recovery, leaving the hospital August 13, sixteen days after operation. She is now enjoying perfect health. As the specimen from this case was not preserved $I$ can not give a report of it.

CASE 2.-Mrs. - , aged 20, had been married three years and had had one labor, no miscarriages.

History.-Menstruation began at 16, regular, not painful, quantity and duration normal. In April, 1904, her periods stopped and Jan. 30, 1905, I delivered her of a healthy baby. Some months later she resumed menstruating and her periods were regular and normal, the last one beginning July 1, 1906 . She missed August 1, but began to flow on August 15. She did not consult me until September 15, having flowed off and on for a month. Vaginal examination revealed nothing $a b$ normal so I prescribed gallic acid, ergotin and hydrastin. This apparently stopped the flow for a few days but it returned and on September 23. I curetted her uterus. I removed a small quantity of what I supposed was hypertrophied endo metrium so made nQ microscopic examination of it. Bimanual palpation showed the fundus to ke high in the pelvis but freely movable, the examination was otherwise negative. A few days later she developed tenderness in the left tubal region and a slight fever which caused me to fear an infection following the curettement as it was done at her home. Hot douches seemed to relieve her and the fever left in a few days.

On Oetober 8, while walking on the street she was taken with severe pain in the left pelvis and fainted; on regaining consciousness she vomited. I found her in collapse and called the ambulance. Reaching home she rapidly recovered from the shock but her left side was rigid and exquisitely tender, so I deferred further examination until morning. The next day I found her with normal temperature and vaginal examination revealed tenderness on the left side and rigidity of the left rectus muscle. I was somewhat chagrined as I had made a diagnosis of ruptured tubal pregnancy and expected to find a mass on the left side; but I could palpate neither tube.

All symptoms gradually subsided except pain referred along the descending colon up to the margin of the ribs. I was very anxious to avoid operative procedure on this patient and decided on the expectant line of treatment.

On October 29, I returned from my vacation and found her

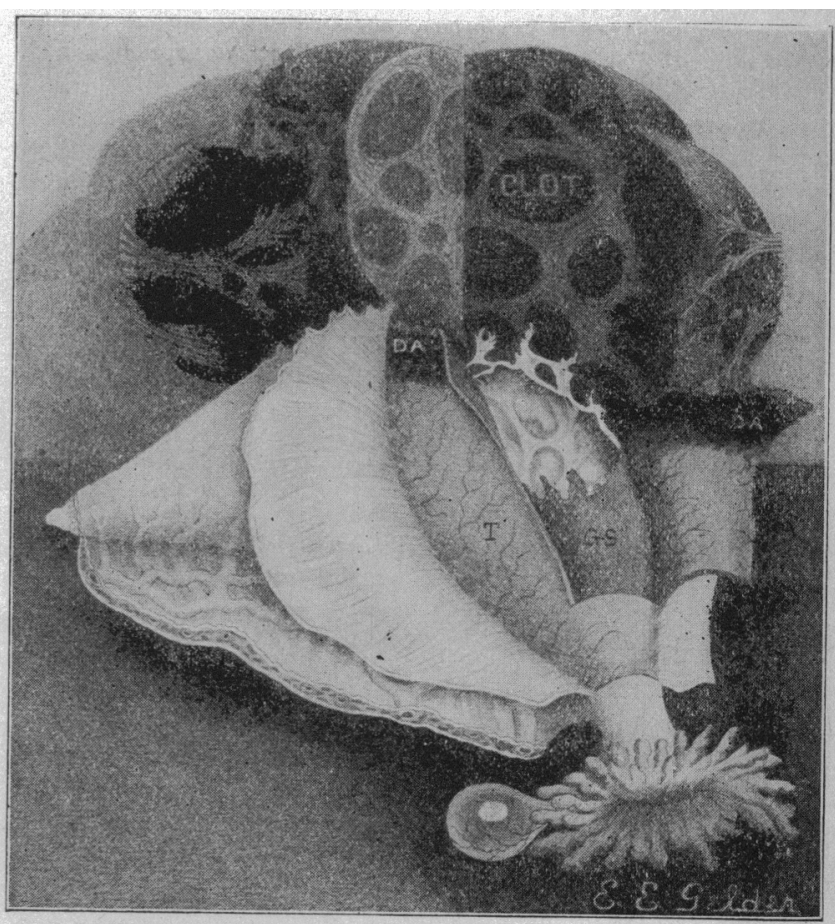

Specimen from Case 2, slightly enlarged. Anterior layer of broad ligament dissected back to show ruptured tube (T). DA. The discolored area along line of rupture. DA flap from the tube is carried to the right to show the ruptured gestation sac (GS), with
Its amniotic lining. Note the small tortuous uterine end of the tube.

with slight fever, pulse 84 , pain along the descending colon becoming worse on urination and defecation, no dulness on percussion, but an increase in resistance on deep palpation just inside the left anterior superior iliac spine. On vaginal examination I detected a mass high in the left pelvis, and exaggerated pulsation in all vessels to the left of the uterus.

Diagnosis.-Ectopic gestation, ruptured high in the pelvis.

Operation.-On Nov. 2, 1906, I opened the abdomen and found free blood in the peritoneal cavity, and a left tubal pregnancy. This had ruptured from the free surface of the tube and become walled off in a pocket formed by the rectum, sigmoid, posterior layer of the broad ligament, and left wall of the pelvis. I removed the clot and left tube. As they were normal I left both ovaries and the right tube. After a thorough cleansing of the pelvis I closed without drainage. The patient enjoyed an afebrile convalescence and left the hospital in good condition eighteen days after operation.

Examination of Specimen.-The uterine end of the tube was small, tortuous, and could not be probed. The covering of the tube was thinned out and torn along an irregular line on 
its convex surface. The latter was congested and markedly discolored along the line of rupture; within was the ruptured gestation sac. No fetus was found. The cause of the ectopic gestation was either a congenital or acquired narrowing of the uterine end of the tube.

\section{SUMMARY.}

1. This was the first conception in Case 1 .

2. Only 18 months had elapsed since the second patient was delivered of her first child.

3. Neither patient had ever presented symptoms of inflammatory or infectious disease of any of the pelvic viscera.

4. Neither had subjective symptoms of pregnancy.

5. In both cases the menstrual period was delayed exactly two weeks.

6. In Case 2 the rupture occurred into the peritoneal cavity and, though no hemostasis was applied, the hemorrhage was not severe.

7. The appearance of physical signs in Case 2 was very late, despite a typical history.

\section{PAINFUL HEEL,}

WITH A DEMONSTRATION OF A THEORY AS TO A MECHANICAL CAUSE.*

JOHN JOSEPH NU'TT, B.L., M.D.

Clinical Instructor in Orthopedic Surgery, Cornell University Medical College: Assistant Surgeon to the New York State Hospital for Crippled and Deformed Children. NEW YORK CITY.

The name which heads this article is a rather unfortunate one, as it is not at all descriptive or distinctive. All pain referred to the plantar surface of the heel is not due to this condition.

Bradford and Lovett say: "This is a condition in which there is a tender and painful area under the middle of the heel." Whitman describes it with calcaneobursitis. Under painful heel he includes all pain referred to the botom of the heel. When the pain is clearly localized he says: "The cause of the symptoms in such cases may be an inflamed bursa lying between the periosteum and the fatty tissue of the heel." Walsham and Hughes do not mention the subject in "Deformities of the Human Foot." Nor does Keetley refer to it in his "Orthopedic Surgery." The French surgeons, however, have recognized this condition, and Duplay has recommended an operation which is endorsed by Whitman. Young's definition, which is clear and exact, is: "Severe pain, accompanied by tenderness, in the center of the heel about the posterior attachment of the plantar fascia."

Painful heel is probably not a rare condition. As the pain is alleviated by rest, the milder cases are usually self-treated and are not seen by the surgeon until complications have arisen which cloak the original disease. Policemen, however, are not able to favor the foot. They can not give it rest even when extremely painful and must seek relief of the physician. Hence its synonym: policeman's heel. The only subjective symptom is the pain. This is constantly in one place. It is excited by walking much more than by standing and is temporarily relieved by absolute rest. If closely questioned the patient may state that the pain is worse just before he brings the foot forward in walking.

* Read before Bellevue Hospital Alumnl Association, June 6, 1906

\section{EXAMINATION.}

Inspection may show nothing abnormal; both feet may be well formed. There will be no external evidence of inflammation. Deep pressure on the spot will usually produce pain. Manipulation will disclose a normal mediotarsal joint and the head of the astragalus and the scaphoid in their normal position. The only abnormality in the uncomplicated cases will be found at the ankle joint. Here the range of flexion will be found to be limited. In other words, non-deforming club-foot exists. If we knew this to be always the case, we could simply class painful heel among the complications of non-deforming club-foot, but doubtless sometimes painful heel, as defined, originates from other causes. Exostosis, bursitis or neuroma may be the primary lesion, though I believe they are more often secondary to the traumatism to the plantar fascia.

\section{A MECHANICAL EXPLANATION.}

A mechanical explanation of a cause of this condition I. wish to demonstrate. As pointed out by Dr. Shaffer when he first described non-deforming club-foot, that condition is usually the cause of flat-foot, and may also cause bunions, corns, calluses, ingrowing toe nails and Morton's disease. He did not mention, however, that painful heel may be a result. As far as I know, painful heel has never been given a mechanical explanation based on a shortened gastrocnemius.

The shortened gastrocnemius is the sine qua non in Shaffer's foot. I use the term "Shaffer's foot" intentionally. Non-deforming club-foot, the name given it by Dr. Shaffer, is more or less confusing to the profession and is repugnant to the laity, especially to the woman who is proud of a well-shaped foot and perhaps a high aristocratic arch. This limitation of dorsal flexion, due to the shortened gastrocnemius, necessitates, in walking, the shifting of the weight of the body to a point more anterior than normal. Consequently an added strain is brought to bear on the plantar fascia and all the plantar tissues.

Normally the superincumbent weight is dispersed through the astragalus in three directions, downward and backward through the calcaneum, downward, forward and outward through the calcaneum, cuboid and two outer metatarsals, and downward, forward and inward through the astragalus, scaphoid, the three cuneiform and the three inner metatarsals.

If the gastrocnemius is but slightly shortened, say dorsal flexion is limited with the foot at a right angle to the leg, then, as the leg is flexed on the foot in locomotion, the weight normally falling on the posterior tubercles of the os calcis is transferred to the other two directions, and the heel, instead of supporting weight, is raised from the ground and strains the plantar fascia. Motion being blocked at the ankle joint, the force is transmitted further along and seeks to obtain movement at the next joint which is the mediotarsal. This extraordinary strain on the plantar fascia weakens it, and, if it elongates, dorsal flexion will take place at the mediotarsal joint and flat-foot will result. When the gastrocnemius is greatly shortened, as when dorsal flexion of the. foot is arrested at 105 degrees or more, with the leg, the weight, in flexing the leg on the foot in the act of walking, is thrown so far forward that the plantar tissues tend rather to become a continuation of the posttibial muscles and the length of the foot is shortened, the plantar fascia becomes contracted and, assisted by 